\title{
Note
}

\section{Use of image analysis to estimate anthocyanin and UV-excited fluorescent phenolic compound levels in strawberry fruit}

\author{
Yosuke Yoshioka*1), Masayoshi Nakayama'), Yuji Noguchi') and Hideki Horie') \\ 1) NARO Institute of Vegetable and Tea Science, 360 Kusawa, Ano, Tsu, Mie 514-2392, Japan \\ 2) NARO Institute of Floricultural Science, 2-1 Fujimoto, Tsukuba, Ibaraki 305-8519, Japan
}

\begin{abstract}
Strawberry is rich in anthocyanins, which are responsible for the red color, and contains several colorless phenolic compounds. Among the colorless phenolic compounds, some, such as hydroxycinammic acid derivatives, emit blue-green fluorescence when excited with ultraviolet (UV) light. Here, we investigated the effectiveness of image analyses for estimating the levels of anthocyanins and UV-excited fluorescent phenolic compounds in fruit. The fruit skin and cut surface of 12 cultivars were photographed under visible and UV light conditions; colors were evaluated based on the color components of images. The levels of anthocyanins and UV-excited fluorescent compounds in each fruit were also evaluated by spectrophotometric and high performance liquid chromatography (HPLC) analyses, respectively and relationships between these levels and the image data were investigated. Red depth of the fruits differed greatly among the cultivars and anthocyanin content was well estimated based on the color values of the cut surface images. Strong UVexcited fluorescence was observed on the cut surfaces of several cultivars, and the grayscale values of the UV-excited fluorescence images were markedly correlated with the levels of those fluorescent compounds as evaluated by HPLC analysis. These results indicate that image analyses can select promising genotypes rich in anthocyanins and fluorescent phenolic compounds.
\end{abstract}

Key Words: anthocyanins, fluorescence, image analysis, strawberry, ultraviolet.

\section{Introduction}

Anthocyanins, which are phenolic compounds, are responsible for the external and internal colors in strawberry (Fragaria $\times$ ananassa Duchesne). Accordingly, anthocyanin content has inevitably been an important selection criterion in strawberry breeding. Anthocyanins have also attracted attention as an important phytochemicals with potential health-promoting effects, such as antioxidative activity (Wang et al. 1997, Wu and Prior 2005). Generally, phytochemicals with potential health-promoting effects include phenolic compounds such as flavonoids, nitrogenous compounds such as chlorophyll derivatives and tocopherols, carotenoids, and ascorbic acids. Of the phenolic compounds, strawberry is abundant in anthocyanins, catechin and flavonols such as quercetin and kaempferol (Hannum 2004). Besides these flavonoids, strawberry fruits are also abundant in several phenolic acids and their derivatives such as ellagic acid (Hannum 2004, Heinonen et al. 1998, Wang and Lin 2000, Zhang et al. 2008). These phenolic compounds are

Communicated by T. Hayashi

Received October 18, 2012. Accepted January 10, 2013.

*Corresponding author (e-mail: ekusoy@affrc.go.jp.) products of plant metabolism that likely serve many functions essential to the growth and survival of the plant; some of them are clearly bioactive in the animals and humans who consume them (Hannum 2004). Recently, in strawberry breeding, as well as other fruit and vegetable, it has become increasingly important to breed new cultivars that are rich in phytochemicals that have potential health benefits, such as antioxidant, anticarcinogenic and antimutagenic effects.

The kinds and amounts of anthocyanins and other phenolic compounds vary widely among existing cultivars (Andersen et al. 2004, Wang and Lin 2000, Yoshida and Tamura 2005), indicating that it may be possible to breed new cultivars that contain higher levels of these compounds. The levels of anthocyanins and other phenolic compounds are usually evaluated by using spectrophotometric and HPLC analyses (Lopes-da-Silva et al. 2002, Wu and Prior 2005). However, these methods require expensive equipment and are time- and labor-intensive to evaluate even a few fruits. Moreover, anthocyanins and other phenolic compound levels vary quantitatively with growing conditions, degree of ripeness and handling after harvest even in the same cultivar. In breeding programs, this means that breeders must evaluate a large amount of fruit throughout the long harvest period to correctly select a promising genotype rich 
in phytochemicals. Therefore, from the breeder's viewpoint, a simplified method to roughly estimate phytochemical composition is desirable to avoid the time and monetary expense of a more accurate evaluation.

The depth of the red color of strawberry fruits is determined by anthocyanins, and the anthocyanin content can be estimated by evaluating fruit color (e.g., Maegawa et al. 1992, Urata et al. 1991, Yoshida et al. 2002). Therefore, more accurate color evaluation could provide a more precise estimate of the anthocyanin content in strawberry fruits. Fruit color has long been quantified by colorimeters. But since the aperture of many colorimeters is limited in size and shape, it is difficult to efficiently and accurately evaluate strawberry fruit that has an uneven surface color, such as skin color with red gradation, existence of green seeds, cut surface color with gradation from red to white. In contrast, digital image analyses can evaluate fruit color more objectively and quantitatively than traditional methods, such as human visual assessment and colorimeters and thus have been successfully used to evaluate plant organ color in other species (e.g., Darrigues et al. 2008, Lootens et al. 2007, Venora et al. 2009, Yoshioka et al. 2004, Yoshioka and Fukino 2010). In addition, imaging techniques under nonvisible light (i.e., infrared and ultraviolet) are well known as efficient tools to detect and evaluate colorless compounds in plant organs. For example, near-infrared imaging techniques have recently been developed to evaluate sugar content in fruit (Martinsen and Schaare 1998, Sugiyama 1999, Tsuta et al. 2002) and ultraviolet imaging techniques can be used to detect some flavonoids and carotenoids in flowers (Fukuta et al. 2005, Nakayama et al. 2006, Sasaki and Takahashi 2002, Yoshioka et al. 2005). Observation under UV-light can also detect UV-excited fluorescent compounds such as phenolic acids (Buschmann et al. 2000, Chaerle et al. 2007, Lichtenthaler and Schweiger 1998) and fluorescent chlorophyll catabolites (Moser et al. 2008, 2009) in plant organs. Therefore, digital image analysis should be useful as a simplified method for estimating anthocyanin content and may have potential applications to the detection and evaluation of colorless compounds in strawberry fruits.

In this study, we demonstrate the effectiveness of image analyses in estimating the levels of anthocyanins and UVexcited fluorescent compounds in strawberry fruits. First, we photographed strawberry fruits of several Japanese strawberry cultivars under visible light, as well as by using UV reflected and UV fluorescence photography. Since color models, the way colors can be represented as typically three or four color components, have different characteristics, the estimate accuracy of the compounds depends on the color components and models used in the image analysis. For example, CIE-Lab color models, that is designed so that the Euclidean distance between color coordinates approximates how well colors are discriminated by the human eye, has three color components: $\mathrm{L}^{*}, \mathrm{a}^{*}$ and $\mathrm{b}^{*}$. Scores of $\mathrm{L}^{*}, \mathrm{a}^{*}$ and $b^{*}$ represent the lightness of the color, position between red/ magenta and green and position between yellow and blue, respectively. Therefore, in order to establish more appropriate estimation methods, we evaluated skin and cut-surface colors on the basis of the color information from three color models: RGB (red, green, blue), HSL (hue, saturation, lightness) and CIE-Lab $\left(L^{*}, a^{*}, b^{*}\right)$. Second, we evaluated anthocyanin and UV-excited fluorescent compound levels by using spectrophotometric and high performance liquid chromatography (HPLC) analyses. Finally, we analyzed the relationships between the compound levels and the image data. We discuss the potential application of image analyses in strawberry breeding programs.

\section{Materials and Methods}

\section{Plant material}

Strawberry fruits of 12 cultivars were obtained from several grocery stores and from the glasshouse at the Institute of Vegetable and Tea Science (Tsu, Mie, Japan) in April 2010 (Table 1). These cultivars were chosen to represent the wide variety of external and internal fruit colors in current cultivars in Japan. We used eight to ten fruits of each cultivar except for 'Sachinoka', which provided 30 fruits from three different lots (Table 1). After removing the calyx, each fruit was cut into two equal parts vertically along the central axis of the fruit. We randomly chose one of each pair of longitudinal halves and used it for the image and spectrophotometric analyses. In addition, of the materials used for the image and spectrophotometric analyses, 51 fruits were subjectively selected so as to cover the variation of UV-excited fluorescence, and used for HPLC analysis to measure fluorescent compound levels.

\section{Image analysis}

For photography under visible light conditions, a handmade lighting chamber was used to provide uniform illumination during image acquisition (Fig. 1). We placed each longitudinally halved fruit in the center of the lighting chamber, and photographed it with or without a cylindrical white Kent paper (approximately $10 \mathrm{~cm}$ in diameter), which was used to convert irradiated light from the two fluorescent lights (Fluorescent High Frequency Linear Light, Edmund Optics Inc., Barrington, NJ, USA) into diffused light. The distance from the camera lens to the fruit was maintained at approximately $60 \mathrm{~cm}$. We photographed both sides of each fruit half (that is, the fruit skin and the cut surface) using a Nikon D70 camera (Nikon Corp., Tokyo, Japan) with a Nikon UV-105 mm F4.5 lens (Tochigi Nikon Corp., Ohtawara, Tochigi, Japan). Images were saved in JPEG file format and converted into BMP format after preprocessing steps such as trimming the image and changing the background color. For fruit skin or cut surface part of each image, we calculated the average red $(R)$, green $(G)$ and blue $(B)$ values of the RGB color model, the hue $(\mathrm{H})$, saturation $(\mathrm{S})$ and lightness (L) values of the HSL color model and the L*, $a^{*}$ and $b^{*}$ values of the CIE-Lab color model as color features of the fruit skin or cut surface of each fruit. 
Table 1. The averages and standard deviations of fourteen lots (twelve cultivars) of CIE-Lab color component values of skin and cut surface images photographed under visible light, of grayscale (Gr) values of cut surface images of UV-excited fluorescent images, and of anthocyanin content evaluated by use of spectrophotometric analysis

\begin{tabular}{|c|c|c|c|c|c|c|c|c|c|}
\hline \multirow{2}{*}{ Cultivar } & \multirow{2}{*}{$\mathrm{n}$} & \multicolumn{3}{|c|}{ Skin } & \multicolumn{4}{|c|}{ Cut surface } & \multirow{2}{*}{ Anthocyanin } \\
\hline & & $\mathrm{L}^{*}$ & $a^{*}$ & $b^{*}$ & $\mathrm{~L}^{*}$ & $a^{*}$ & $\mathrm{~b}^{*}$ & $\mathrm{Gr}$ & \\
\hline Akihime & 10 & $45.8 \pm 3.8^{\mathrm{a}}$ & $55.1 \pm 2.9^{\mathrm{ab}}$ & $33.3 \pm 4.4^{a b}$ & $5.6 \pm 4.1 \mathrm{bcd}$ & $18.2 \pm 6.2 \mathrm{def}$ & $8.0 \pm 2.2^{f}$ & $13.9 \pm 2.6$ defg & $0.053 \pm 0.017$ ef \\
\hline Amaou & 10 & $5.6 \pm 2.6^{\mathrm{fg}}$ & $49.0 \pm 2.6^{\text {cde }}$ & $20.3 \pm 4.0^{f}$ & $.4 \pm 2.4^{\mathrm{h}}$ & $45.0 \pm 2.8^{\mathrm{a}}$ & $25.5 \pm 2.0^{\mathrm{a}}$ & $4.6 \pm 0.8^{\mathrm{g}}$ & $0.163 \pm 0.020^{\mathrm{a}}$ \\
\hline Asukaruby & 10 & $41.0 \pm 3.1 \mathrm{bcd}$ & $53.4 \pm 3.6 \mathrm{abc}$ & $25.7 \pm 5.4^{\mathrm{cdef}}$ & $1 \pm 5.4^{g}$ & $30.6 \pm 5.0^{\mathrm{b}}$ & $11.0 \pm 3.2 \mathrm{def}$ & $19.8 \pm 6.5^{\mathrm{cdef}}$ & $0.089 \pm 0.028^{\mathrm{cd}}$ \\
\hline Benihoppe & 10 & $41.2 \pm 3.1 \mathrm{bcd}$ & $48.8 \pm 3.0$ cde & $27.9 \pm 3.4$ bcde & $75.6 \pm 3.2 \mathrm{bcd}$ & $19.5 \pm 5.6^{\text {cde }}$ & $12.7 \pm 2.6^{\text {cde }}$ & $18.1 \pm 3.9 \mathrm{cdefg}$ & $0.072 \pm 0.019 \mathrm{de}$ \\
\hline Hinoshizuku & 10 & $39.7 \pm 2.2 \mathrm{def}$ & $52.3 \pm 1.4 \mathrm{bc}$ & $25.9 \pm 3.0^{\text {cdef }}$ & $70.0 \pm 4.4 \mathrm{def}$ & $25.7 \pm 3.2 \mathrm{bc}$ & $9.5 \pm 1.6^{\mathrm{ef}}$ & $22.4 \pm 7.8$ bcdef & $0.089 \pm 0.013^{\mathrm{cd}}$ \\
\hline Kaorino & 10 & $44.3 \pm 2.7 \mathrm{abc}$ & $54.8 \pm 2.6^{\mathrm{ab}}$ & $30.9 \pm$ & $.3 \pm 2.1^{\mathrm{ab}}$ & $12.6 \pm 4.0^{\mathrm{fg}}$ & $9.8 \pm 2.1$ def & $28.7 \pm 9.1^{\mathrm{abc}}$ & $0.042 \pm 0.014^{\mathrm{f}}$ \\
\hline Karenberry & 10 & $40.3 \pm 1.3$ cde & $49.1 \pm 2.3$ cde & $23.0 \pm 3$ & $64.7 \pm 4.9^{\mathrm{fg}}$ & $30.6 \pm 1.9^{b}$ & $13.1 \pm 1.0^{\mathrm{cd}}$ & $12.5 \pm 1.6^{\mathrm{efg}}$ & $0.094 \pm 0.013^{\mathrm{cd}}$ \\
\hline Nyoho & 8 & $37.8 \pm 0.8^{\operatorname{defg}}$ & $51.3 \pm 1.1 \mathrm{bcd}$ & $23.0 \pm 1.3$ def & $68.9 \pm 2.0^{\text {efg }}$ & $28.8 \pm 1.7^{b}$ & $12.8 \pm 1.5^{\text {cde }}$ & $18.7 \pm 2.2^{\mathrm{cdefg}}$ & $0.094 \pm 0.005$ bcd \\
\hline Sachinoka & 10 & $37.0 \pm 3.1 \mathrm{defg}$ & $50.5 \pm 3.4$ bcde & $26.0 \pm 5.3^{\mathrm{cdef}}$ & $69.9 \pm 5.6^{\text {ef }}$ & $25.9 \pm 7.9 \mathrm{bc}$ & $14.4 \pm 3.4^{\mathrm{c}}$ & $40.4 \pm 22.2^{\mathrm{a}}$ & $0.108 \pm 0.019 \mathrm{bc}$ \\
\hline Sachinoka & 10 & $33.8 \pm 2.7^{\mathrm{g}}$ & $46.2 \pm 4.1^{\mathrm{e}}$ & $20.5 \pm 5.1^{\mathrm{f}}$ & $68.2 \pm 2.8^{\text {efg }}$ & $29.2 \pm 4.0^{b}$ & $18.1 \pm 2.5^{b}$ & $27.9 \pm 18.4$ abcd & $0.124 \pm 0.015^{b}$ \\
\hline Sachinoka & 10 & $36.2 \pm 4.1 \mathrm{efg}$ & $46.8 \pm 4.4 \mathrm{de}$ & $21.6 \pm 6.8$ ef & $69.5 \pm 2.2 \mathrm{efg}$ & $28.9 \pm 3.0^{b}$ & $15.3 \pm 1.4 \mathrm{bc}$ & $36.2 \pm 9.0^{\mathrm{ab}}$ & $0.115 \pm 0.029 \mathrm{bc}$ \\
\hline Sagahonoka & 10 & $47.1 \pm 2.5^{\mathrm{a}}$ & $57.8 \pm 1.8^{\mathrm{a}}$ & $37.8 \pm 1.9^{\mathrm{a}}$ & $83.1 \pm 2.0^{\mathrm{a}}$ & $7.3 \pm 2.8^{g}$ & $7.8 \pm 1.5^{f}$ & $24.5 \pm 3.4$ bcde & $0.033 \pm 0.008^{f}$ \\
\hline Sanukihime & 10 & $45.0 \pm 3.5 \mathrm{ab}$ & $50.0 \pm 3.9$ cde & $29.9 \pm 3.9 \mathrm{bcd}$ & $76.8 \pm 3.2 \mathrm{bc}$ & $15.5 \pm 4.5$ ef & $9.6 \pm 2.4$ ef & $9.7 \pm 1.8^{\mathrm{fg}}$ & $0.060 \pm 0.019$ ef \\
\hline Tochiotome & 10 & $39.0 \pm 3.4 \mathrm{def}$ & $47.0 \pm 3.7 \mathrm{de}$ & $21.3 \pm 5.5^{f}$ & $72.0 \pm 3.7 \mathrm{cde}$ & $24.7 \pm 3.8 \mathrm{bcd}$ & $13.2 \pm 1.8^{\mathrm{cd}}$ & $19.1 \pm 5.2^{\text {cdef }}$ & $0.093 \pm 0.026^{\mathrm{cd}}$ \\
\hline Ave & & 40.3 & 50.9 & 26.2 & & 24.5 & 12.9 & 21.2 & 0.088 \\
\hline C.V. & & 0.10 & 0.07 & 0.20 & 0.10 & 0.38 & 0.36 & 0.47 & 0.39 \\
\hline
\end{tabular}

n, number of fruits; C.V., Coefficient of variation.

Lots (cultivars) labeled with different letters differed significantly at $p<0.05$, Tukey-Kramer multiple-comparison test.

A
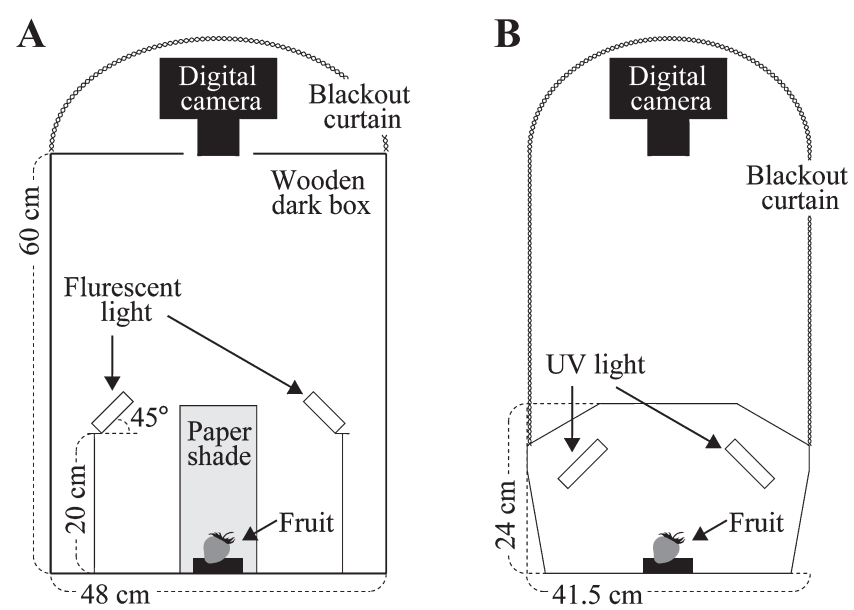

Fig. 1. Schematic diagrams of the photographic equipment under (A) visible light and (B) ultraviolet (UV) light.

For photography under ultraviolet light conditions, a lighting chamber (CSN-15AC/AC, Cosmo Bio Co., Ltd., Tokyo, Japan) consisting of two black light (UV) lamps (CST-15A, Cosmo Bio Co., Ltd., Tokyo, Japan) that emitted near-UV rays (around $365 \mathrm{~nm}$ ) was used. The fruit skin and cut surface were photographed through several different types of filters mounted on the Nikon UV-105 mm F4.5 lens fitted to the Nikon D70. The filters used were a band-pass filter (U-360, Hoya Corp., Tokyo Japan) that transmits ultraviolet light and absorbs visible light and long-pass filters (GG455, GG475, GG495 and OG515, Schott AG, Mainz, Germany) that attenuated shorter wavelengths and transmitted longer wavelengths over the active range of the target spectrum. For example, the stopband limit $\lambda_{\mathrm{s}}$ (specified at
$0.001 \%$ internal transmittance), cut-off position $\lambda_{\mathrm{s}}$ (specified at $50 \%$ internal transmittance) and pass-band limit $\lambda_{p}$ (specified at $92 \%$ internal transmittance for this filter) of GG475 are $410 \mathrm{~nm}, 475 \pm 6 \mathrm{~nm}$ and $550 \mathrm{~nm}$, respectively. All necessary adjustments, such as focus control and f-stop, were determined under visible light conditions in a pilot study. Images were saved in JPEG file format and converted into BMP format after preprocessing steps such as trimming and changing the background color. Since the strength of blue fluorescence can be represented by grayscale values $(\mathrm{Gr}=0.299 \mathrm{R}+0.587 \mathrm{G}+0.114 \mathrm{~B})$, average grayscale values (Gr) for the fruit skin and cut surface were calculated as color features.

\section{Spectrophotometric and HPLC analyses}

Anthocyanin and fluorescent compound levels per unit weight of strawberry fruit were measured by means of spectrophotometry and HPLC, respectively, based on the following simplified methods. Each longitudinally halved fruit was weighed and finely crushed with a plastic stirring rod in a 250-mL plastic bottle. Each sample (at $x \mathrm{~g}$ ) was extracted with a solvent (at $9 \times x \mathrm{~mL}$ ), methanol : formic acid : water $(19: 1: 20 \mathrm{v} / \mathrm{v} / \mathrm{v})$, in a refrigerator for $24 \mathrm{~h}$. After visual confirmation that the solid residue had decolorized almost completely, the supernatant $(25 \mathrm{~mL})$ was placed in polypropylene centrifugation tubes and centrifuged at 10,000 rpm for 10 minutes at $15^{\circ} \mathrm{C}$. The filtrates were then diluted 10 -fold with distilled water. The absorption of each diluted filtrate was measured spectrophotometrically at $510 \mathrm{~nm}$ (U-2810, Hitachi High-Technologies Corp., Tokyo, Japan). The HPLC analysis was performed on an LC10-vp system (Shimadzu, Kyoto, Japan) with a fluorimetric detector. 
Twenty $\mu \mathrm{L}$ of each diluted filtrate was injected into a Mightysil RP-18GP (Kanto Chemical Co., Inc., Tokyo, Japan) column $(5 \mu \mathrm{m}, 150 \times 4.6 \mathrm{~mm})$, which was eluted with a gradient of $1 \%$ acetic acid (A) and $90 \%$ acetonitrile (B) at a flow rate of $1 \mathrm{~mL} / \mathrm{min}$. The gradient profile used was 0 $2 \mathrm{~min}$, isocratic $0 \% \mathrm{~B}, 2-42 \mathrm{~min} .0 \%-20 \% \mathrm{~B}$ and $42-$ $45 \mathrm{~min}$. isocratic $20 \% \mathrm{~B}$. The signals were monitored through excitation and emission wavelengths at $360 \mathrm{~nm}$ and $450 \mathrm{~nm}$, respectively.

\section{Statistical analyses}

Differences between pairs of cultivars (or lots) in terms of color features, anthocyanin content and fluorescent compound content were examined by using the Tukey-Kramer multiple-comparison procedure. Correlations between color components and anthocyanins levels were analyzed by using Pearson's product-moment correlation analysis using averages of cultivars (or lots). To obtain the best set of parameters (color components) for predicting anthocyanin content, we conducted stepwise multiple regression analyses separately for each color model (i.e., RGB, HSL and CIE-Lab) using average values for the cultivars (or lots). Forward selection of independent variables was performed by using a significance level of 5\%. Relationship between the Gr values for the UV-excited fluorescence images and fluorescent compounds level was also analyzed by correlation analysis. All statistical analyses were performed using the JMP 8.0 software (SAS Institute Inc., Cary, NC, USA).

\section{Results}

Skin and cut surface colors differed greatly among cultivars (or lots) under visible light (Fig. 2A, 2C). Since both the skin and the cut surface of strawberry fruit strongly reflect light, placement of the white Kent paper on the space between the light source and the object was very helpful when evaluating the color (Fig. 2A). The UV absorption (or reflection) of strawberry skin did not differ among cultivars (Fig. 2B), and UV-excited fluorescence was not observed for all cultivars (figure not shown). In contrast, the UV absorption (or reflection) of the strawberry's cut surface varied widely among cultivars (Fig. 2D) and strong UV-excited fluorescence was observed for several cultivars (Fig. 2E, 2F and Table 1). Fig. $2 \mathrm{E}$ and $2 \mathrm{~F}$ shows cut surface images under ultraviolet light with long-pass filters GG475 and GG495 respectively. These two images were similar to those with other two filters, GG455 and OG515 (figure not shown). The cut surface area that absorbed UV light corresponded to the redpigmented area, where anthocyanins were present (Fig. 2C, 2D). The area that did not excite fluorescence also tended to correspond to the red-pigmented area, whereas the white area, which has little to no anthocyanins, did not necessarily excite fluorescence (Fig. 2E, 2F). For example, the central part of the cut surface of the 'Sagahonoka' and 'Akihime' cultivars tended to be white, but did not excite fluorescence strongly (Fig. 2E, 2F). In contrast, the white central part of

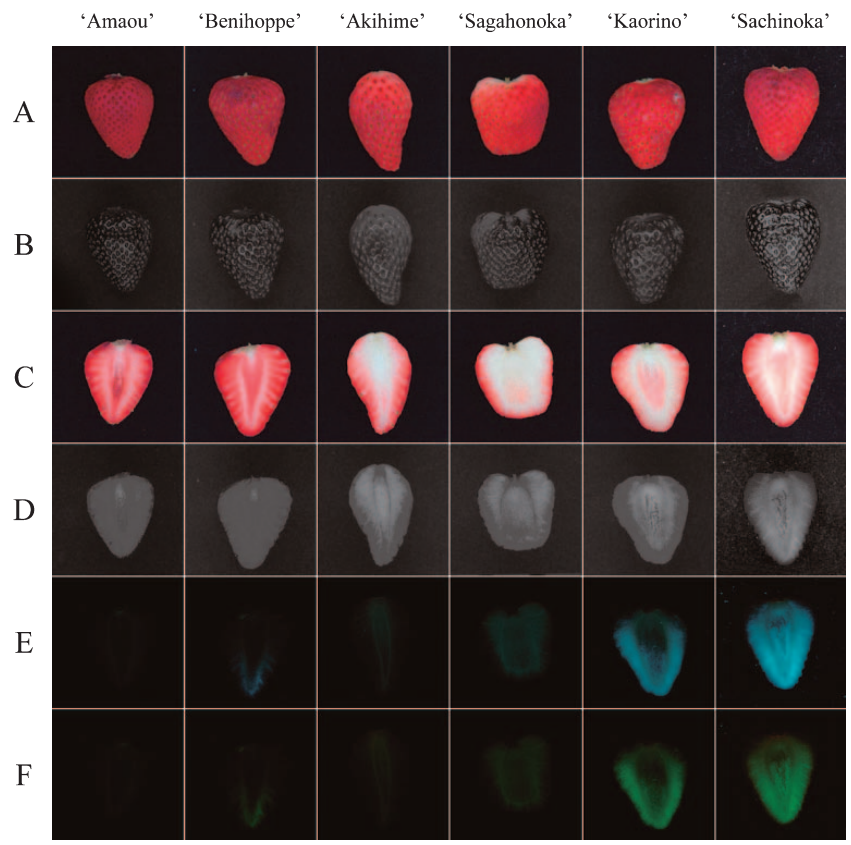

Fig. 2. Examples of skin and cut surface images of seven cultivars photographed in several ways: (A) skin images under visible light with a cylindrical white Kent paper; (B) skin images under ultraviolet light with a band-pass filter that transmits ultraviolet light and absorbs visible light; (C) cut surface images under visible light with a cylindrical white Kent paper, (D) cut surface images under ultraviolet light with a band-pass filter that transmits ultraviolet light and absorbs visible light, and with long-pass filters (E) GG475 and (F) GG495 that attenuate shorter wavelengths and transmit longer wavelengths over the active range of the target spectrum.

the 'Sachinoka' and 'Kaorino' cultivars exhibited strong fluorescence (Fig. 2E, 2F). In addition, in some cultivars, such as 'Sachinoka' the edges of the fruit, which were moderately red, excited fluorescence strongly (Fig. 2E, 2F). For further image analyses, we used the following images that differed among the cultivars: skin and cut surface images photographed under visible light conditions with white Kent paper (Fig. 2A, 2C) and UV-excited fluorescence images photographed with a GG475 filter (Fig. 2E).

In the visible images there were significant differences between cultivars (or lots) for all of the color component values of the CIE-Lab (Table 1), RGB and HSL (data not shown) color models. The coefficients of variation (CVs) for these color components in the skin and cut surface ranged from 0.02 for the $H$ value to 0.20 for the $b^{*}$ value and from 0.02 for the $\mathrm{R}$ value to 0.38 for the $\mathrm{a}^{*}$ value, respectively. For most of the color components, the CVs for the cut surface were larger than those for the skin, and the $a^{*}$ and $b^{*}$ values were particularly high for the cut surface (Table 1). Anthocyanin content, evaluated by means of spectrophotometric analysis, also differed significantly among cultivars (or lots) and its CV was higher than that of any color component (Table 1).

Significant correlations were observed between color 
A

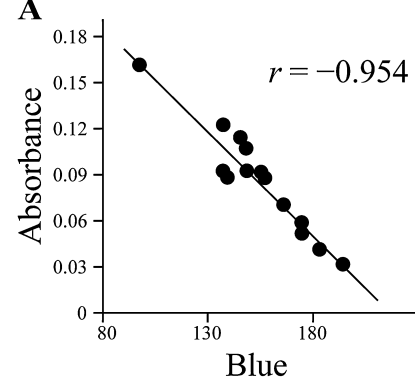

C

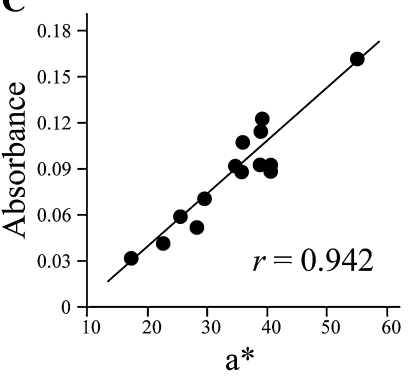

B

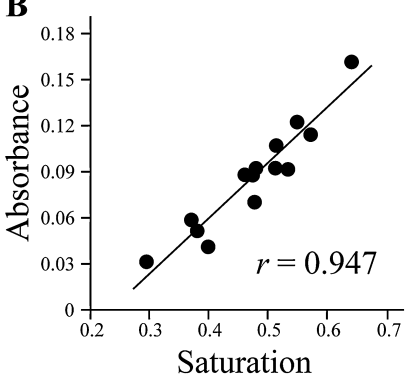

D

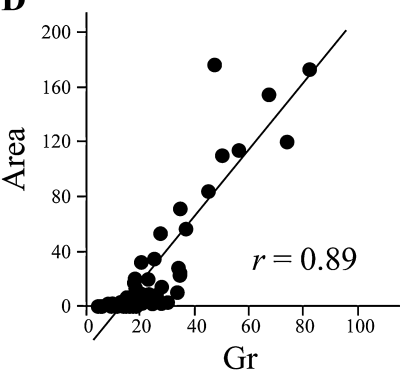

A

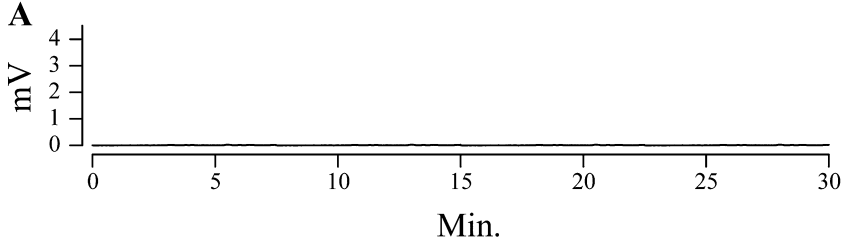

B

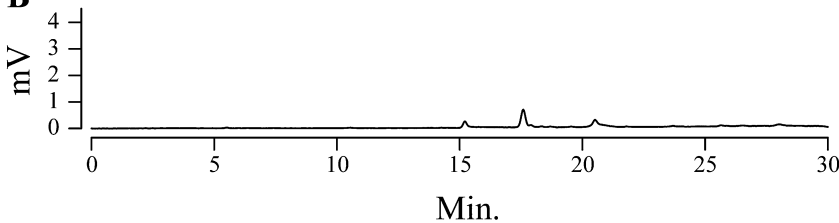

C

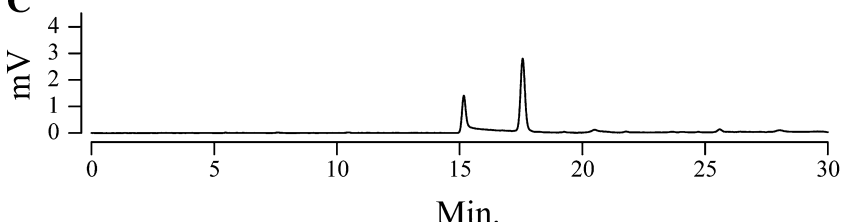

D

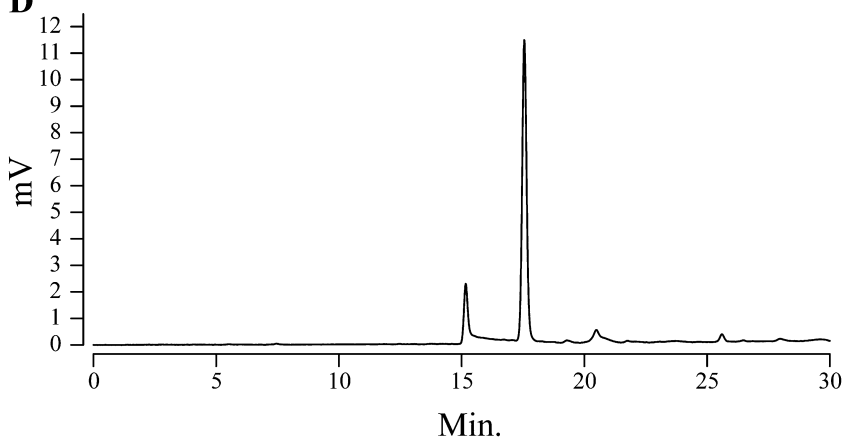

Fig. 4. HPLC chromatograms of UV-excited fluorescent compounds of (A) non-, (B) weakly, (C) intermediately and (D) strongly fluorescent fruits.

strongly UV-excited fluorescent fruits. The chromatograms of UV-excited fluorescent fruits had two relatively strong peaks at retention times of near 15 and $17 \mathrm{~min}$. The first eluting peak was smaller than the second, especially for strongly UV-excited fluorescent fruit (Fig. 4D). The areas of the two peaks were then summed and the resultant value used as the level of the fluorescent compounds. As expected, the Gr values for the UV-excited fluorescence images were significantly correlated with the levels of fluorescent compounds (Fig. 3D).

\section{Discussion}

The red color of strawberry fruit is essentially determined by anthocyanins and as a result, breeders and researchers can estimate anthocyanin levels by visual assessment and colorimeters (e.g., Maegawa et al. 1992, Urata et al. 1991, Yoshida et al. 2002). Therefore, it can easily be seen how image analysis of the red depth of fruit could provide more objective and accurate estimates of anthocyanin content. However, there was little research to support clear and images (Table 1). Fig 4 shows a typical HPLC chromatogram of extracts from non-, weakly, intermediately and 
Table 2. Estimation of parameters in three regression models for anthocyanin content evaluated by using spectrophotometric analysis

\begin{tabular}{|c|c|c|c|c|}
\hline Variable & Estimate & SE & $t$ value & $\operatorname{Pr}>|t|$ \\
\hline \multicolumn{5}{|c|}{$\operatorname{RGB}\left(R^{2}=0.978, F=150.12, p<0.0001\right)$} \\
\hline Intercept & 0.4214 & 0.0286 & 14.71 & $<0.0001$ \\
\hline Cut surface B & -0.0010 & 0.0001 & -8.76 & $<0.0001$ \\
\hline Skin R & -0.0007 & 0.0002 & -4.37 & 0.0014 \\
\hline Skin B & -0.0011 & 0.0005 & -2.24 & 0.0488 \\
\hline \multicolumn{5}{|c|}{$\operatorname{HSL}\left(R^{2}=0.948, F=100.46, p<0.0001\right)$} \\
\hline Intercept & 0.1368 & 0.0683 & 2.00 & 0.0704 \\
\hline Cut surface $S$ & 0.2485 & 0.0433 & 5.74 & 0.0001 \\
\hline Cut surface L & -0.2287 & 0.0697 & -3.28 & 0.0073 \\
\hline \multicolumn{5}{|c|}{ CIE-Lab $\left(R^{2}=0.980, F=160.09, p<0.0001\right)$} \\
\hline Intercept & 0.1334 & 0.0357 & 3.74 & 0.0038 \\
\hline Cut surface $a^{*}$ & 0.0016 & 0.0003 & 4.83 & 0.0007 \\
\hline Cut surface $b^{*}$ & 0.0022 & 0.0006 & 3.41 & 0.0067 \\
\hline Skin L & -0.0028 & 0.0007 & -3.96 & 0.0027 \\
\hline
\end{tabular}

strong relationships between image information and pigment content in the strawberry. In this study, we successfully demonstrated the usefulness of a simple method to estimate anthocyanin levels based on image analyses. Using this method, we found that the cut surface (flesh) color was a more effective estimator than was skin color. Yoshida and Tamura (2005) revealed that the difference in the concentration of anthocyanins among cultivars was larger in inner flesh than in skin. Similarly, there was wider variation in cut surface color than in skin color among our samples (Table 1), particularly cultivars whose inside does not turn red, such as 'Sagahonoka' and 'Kaorino' (Fig. 2C). The wider variation would partly result in superiority of the cut surface colors as estimators of anthocyanin levels. Anthocyanin content was efficiently estimated by using a single color component of each color model, such as the blue of the RGB model, saturation of the HSL model, or a* of the CIE-Lab color model in the cut surface image (Fig. 3). However, analysis of more than one color component of the skin or cut surface images improved the accuracy of the estimation of anthocyanin content (Table 2). No one color model was far superior to the others, indicating that any of the three color models could be used to estimate anthocyanin content.

The cut surface of the strawberry fruits of some cultivars emits fluorescence under UV light, indicating that the fruits of these cultivars bear fluorescent compounds in their internal parts. Previous studies have shown that the UV-induced blue-green fluorescence emission of banana fruit is due to chlorophyll catabolites (Moser et al. 2008, 2009). Similar fluorescence emission observed from the leaves of many species is thought to be primarily due to hydroxycinammic acid derivatives (that is, phenolic acid compounds) such as ferulic acid, p-coumaric acid and caffeic acid and to flavonoids such as kaempferol and quercetin (Lichtenthaler and Schweiger 1998, Morales et al. 1996). In the case of the strawberry, the kind and the amount of compounds in the fruit (Määttä-Riihinen et al. 2004, Mattila et al. 2006, Zhang et al. 2008) and their light-absorbing characteristics suggest that hydroxycinnamic acid derivatives, especially coumaric acid and its glycosides, are most likely responsible for the blue-green fluorescence. The hydroxycinnamic acid derivatives are well known to be associated with antioxidant activity and other human health benefits (Balasundram et al. 2006, Szajdek and Borowska 2008). The significant correlation between the fluorescent signal strength and the level of the phenolic acid compounds, as determined by HPLC analysis, indicates that UV-excited fluorescence photography techniques and image analysis will be useful tools to obtain information about phenolic acid compound levels and their distribution within a fruit.

Optical instruments such as digital cameras and scanners are now commonplace and it is easy to acquire digitized photographs. In fact, many breeders photograph their breeding materials and store the images on their computers. In many cases, the purpose of photography has been limited to recording plant part appearance and image data have not been widely used for other purposes. However, we can expand the use of image data if we modify the photographic techniques and environments slightly. Our approach not only provides more objective and quantitative evaluation of red color compared with human visual assessment, but it also allows estimates of levels of anthocyanins and colorless phenolic acids that are invisible to the naked eye. These days, it is hard to argue with the health benefits of a diet rich in vegetables and fruits, and those that contain large amounts of phenolic compounds such as flavonoids and phenolic acids have enhanced marketability. Therefore, we anticipate that breeders will have an increased need to evaluate the phenolic compound content of the strawberries in their breeding programs. The estimation methods described here have the potential to replace lengthy lab work and expensive lab equipment with rapid image analyses and relatively inexpensive computers and optical instruments. From a longrange viewpoint, this should promote efficiency and reduce costs in breeding programs. Therefore, the image analyses described here represent useful tools for evaluating genotypes rich in phenolic compounds and for selecting promising genotypes in strawberry breeding.

\section{Acknowledgments}

We thank the Fukuoka Agricultural Research Center, Saga Prefectural Agricultural Research Center and Shizuoka Prefectural Research Institute of Agriculture and Forestry for providing seedlings, and Emi Kitadani (NARO), Nobuko Fukino (NARO) and Takaharu Kameoka (Mie University) for their technical advice. This work was supported by KAKENHI (20880035).

\section{Literature Cited}

Andersen,Ø.M., T.Fossen, K.Torskangerpoll, A. Fossen and U.Hauge 
(2004) Anthocyanin from strawberry (Fragaria ananassa) with the novel aglycone, 5-carboxypyranopelargonidin. Phytochemistry 65: 405-410.

Balasundram, N., K.Sundram and S.Samman (2006) Phenolic compounds in plants and agri-industrial by-products: Antioxidant activity, occurrence, and potential uses. Food Chem. 99: 191-203.

Buschmann,C., G.Landgsdorf and H.K.Lichtenthaler (2000) Imaging of the blue, green, and red fluorescence emission of plants: an overview. Photosynthetica 38: 483-491.

Chaerle, L., S.Lenk, D. Hagenbeek, C.Buschmann and D.Van Der Straeten (2007) Multicolor fluorescence imaging for early detection of the hypersensitive reaction to tobacco mosaic virus. J. Plant Physiol. 164: 253-262.

Darrigues,A., J.Hall, E.van der Knaap, D.M.Francis, N.Dujmovic and S.Gray (2008) Tomato analyzer-color test: a new tool for efficient digital phenotyping. J. Am. Soc. Hortic. Sci. 133: 579-586.

Fukuta, N., M. Miyasaka, R. Saito, K. Kuchitsu and M. Nakayama (2005) The relationship between characteristics of optical image under UV light and flavonoid content in acyanic petals of Eustoma grandiflorum. Hort. Res. 4: 147-151.

Hannum, S.M. (2004) Potential impact of strawberries on human health: a review of the science. Crit. Rev. Food Sci. Nutr. 44: 1-17.

Heinonen,I.M., A.S.Meyer and E.N.Frankel (1998) Antioxidant activity of berry phenolics on human low-density lipoprotein and liposome oxidation. J. Agric. Food Chem. 46: 4107-4112.

Lichtenthaler,H.K. and J.Schweiger (1998) Cell wall bound ferulic acid, the major substance of the blue-green fluorescence emission of plants. J. Plant. Physiol. 152: 272-282.

Lootens,P., J.van Waes and L.Carlier (2007) Evaluation of the tepal colour of Begonia $\times$ tuberhybrida Voss. for DUS testing using image analysis. Euphytica 155: 135-142.

Lopes-da-Silva,F., S. de Pascual-Teresa, J.Rivas-Gonzalo and C. Santos-Buelga (2002) Identification of anthocyanin pigments in strawberry (cv Camarosa) by LC using DAD and ESI-MS detection. Eur. Food Res. Technol. 2002: 248-253.

Määttä-Riihinen,K.R., A.Kamal-Eldin and R.Törrönen (2004) Identification and quantification of phenolic compounds in berries of Fragaria and Rubus species (family Rosaceae). J. Agric. Food Chem. 52: 6178-6187.

Maegawa,H. (1992) Studies on fruit coloring in strawberry [Fragaria ananassa] cv. "Toyonoka", 1: Varietal differences in characteristics of coloration and changes in color development of fruit surfaces during maturation. Bull. Nara Agric. Exp. Sta. 23: 13-20.

Martinsen,P. and P.Schaare (1998) Measuring soluble solids distribution in kiwifruit using near-infrared imaging spectroscopy. Postharvest Biol. Technol. 14: 271-281.

Mattila,P., J.Hellström and R.Törrönen (2006) Phenolic acids in berries, fruits, and beverages. J. Agric. Food Chem. 54: 7193-7199.

Morales,F., Z.G.Cerovic and I.Moya (1996) Time-resolved blue-green fluorescence of sugar beet (Beta vulgaris L.) leaves. Spectroscopic evidence for the presence of ferulic acid as the main fluorophore of the epidermis. Biochim. Biophys. Acta 1273: 251-262.

Moser,S., T.Müller, M.O.Ebert, S.Jockusch, N.J.Turro and B.Kräutler (2008) Blue luminescence of ripening bananas. Angew. Chem. Int Ed. 47: 8954-8957.

Moser,S., T.Müller, A.Holzinger, C.Lütz, S.Jockusch, N.J.Turro and
B.Kräutler (2009) Fluorescent chlorophyll catabolites in bananas light up blue halos of cell death. Proc. Natl. Acad. Sci. USA 106: 15538-15543.

Nakayama,M., M.Miyasaka, T.Maoka, M.Yagi and N.Fukuta (2006) A carotenoid-derived yellow Eustoma screened under blue and ultraviolet lights. J. Jpn. Soc. Hort. Sci. 75: 161-165.

Sasaki,K. and T.Takahashi (2002) A flavonoid from Brassica rapa flower as the UV-absorbing nectar guide. Phytochem. 61: 339343 .

Sugiyama,J. (1999) Visualization of sugar content in the flesh of a melon by near-infrared-imaging. J. Agric. Food Chem. 47: 27152718

Szajdek, A. and E.J.Borowska (2008) Bioactive compounds and health-promoting properties of berry fruits: A review. Plant Foods Hum. Nutr. 63: 147-156.

Tsuta,M., J.Sugiyama and Y.Sagara (2002) Near-infrared imaging spectroscopy based on sugar absorption band for melons. J. Agric. Food Chem. 50: 48-52.

Urata,J., T.Tanaka and T.Matuo (1991) The effect of environmental factors on the fruit color of strawberry [Fragaria ananassa] CV. Toyonoka. Bull. of the Saga Pref. Agric. Exp. Sta. 27: 61-72.

Venora, G., O.Grillo, C.Ravalli and R.Cremonini (2009) Identification of Italian landraces of bean (Phaseolus vulgaris L.) using an image analysis system. Sci. Hortic. 121: 410-418.

Wang,H., G.Cao and R.L.Prior (1997) Oxygen radical absorbing capacity of anthocyanins. J. Agric. Food. Chem. 45: 304-309.

Wang, S.Y. and H.S.Lin (2000) Antioxidant activity in fruits and leaves of blackberry, rasberry, and strawberry varies with cultivar and developmental stage. J. Agric. Food Chem. 48: 140-146.

$\mathrm{Wu}, \mathrm{X}$. and R.L.Prior (2005) Systematic identification and characterization of anthocyanins by HPLS-ESI-MS/MS in common foods in the United States: fruits and berries. J. Agric. Food Chem. 53: 2589-2599.

Yoshida,Y., N.Koyama and H.Tamura (2002) Color and anthocyanin composition of strawberry fruit: changes during fruit development and differences among cultivars, with special reference to the occurrence of pelargonidin 3-malonylglucoside. J. Jpn. Soc. Hort. Sci. 71: 355-361.

Yoshida,Y. and H.Tamura (2005) Variation in concentration and composition of anthocyanins among strawberry cultivars. J. Jpn. Soc. Hort. Sci. 74: 36-41.

Yoshioka, Y., H.Iwata, R. Ohsawa and S.Ninomiya (2004) Quantitative evaluation of flower colour pattern by image analysis and principal component analysis of Primula sieboldii E. Morren. Euphytica 139: 179-186.

Yoshioka, Y., A.Horisaki, K. Kobayashi, Syafaruddin, S.Niikura, S. Ninomiya and R. Ohsawa (2005) Intraspecific variation in the ultraviolet colour proportion of flowers in Brassica rapa L. Plant Breed. 124: 551-556

Yoshioka, Y. and N.Fukino (2010) Image-based phenotyping: use of colour signature in evaluation of melon fruit colour. Euphytica 171: 409-416.

Zhang,Y., N.P.Seeram, R.Lee, L.Feng and D.Heber (2008) Isolation and identification of strawberry phenolics with antioxidant and human cancer cell antiproliferative properties. J. Agric. Food Chem. 56: 670-675. 